\title{
Magnetic Domain Structure and Magnetically-Induced Reorientation in Ni-Mn-Ga Magnetic Shape Memory Alloy
}

\author{
O. HECZKO* AND V. BRADSHAW
}

Dep. of Functional Materials, Institute of Physics, CAS, Na Slovance 2, 18228 Prague 8, Czech Republic

\begin{abstract}
Magnetization process during magnetically induced reorientation and related magnetic domains of cuboid $\mathrm{Ni}_{50} \mathrm{Mn}_{28.5} \mathrm{Ga}_{21.5}$ single crystal with $\{100\}$ faces was investigated. Magnetic domains were visualized using magneto-optical indicator. The domains pattern is determined by strong uniaxial magnetic anisotropy of NiMn-Ga martensite. Thanks to magnetically induced reorientation the domains arrangements for all three crystal orientations could be obtained and we showed that the size of domains scales with square root of the thickness and penetrates through whole crystal. Visualization of magnetic domains on all faces of cuboid provides the 3D model of magnetic domains.
\end{abstract}

DOI: 10.12693/APhysPolA.131.1063

PACS/topics: 75.50.Cc, 75.60.Ch, 75.80.+q

\section{Introduction}

The Heusler alloys Ni-Mn-Ga belong to the class of material exhibiting reversible martensitic transformation from cubic austenite to low symmetry martensite. As a consequence the material exhibits various shape memory effects. Moreover, the compounds close to stoichiometric $\mathrm{Ni}_{2} \mathrm{MnGa}$ are ferro- or ferrimagnetic at room temperature and can exhibit so-called magnetic shape memory effects [1]. Thanks to the difference between saturation magnetization between phases the materials exhibit magnetically induced transformation, i.e. the martensitic transformation temperature can be shifted by magnetic field in direct analogy with stress-induced transformation in classical shape memory materials.

More importantly these materials also exhibit giant magnetic-field-induced-strain up to $12 \%[2,3]$, which is called magnetically induced reorientation (MIR) [1]. This effect occurs thanks to extremely high mobility of twin boundaries (ferroelastic interfaces) and no phase transformation is involved. The twin boundary separates two ferroelastic domains or twin variants with different orientations. By moving these twin boundaries the ferroelastic domain (or variant) favourably oriented in magnetic field grows on the expenses of other variants. Since the twin boundary forms elastically compatible interface the movement of boundaries is highly reversible [4]. The extremely high mobility of twin boundaries may be connected with structure instability of $\mathrm{Ni}-\mathrm{Mn}-\mathrm{Ga}$ and complex structure of twinning [5]. It is not quite clear if ferromagnetism can play some role in the mobility. Due to structural arrangement the mobile twin boundary between variants is also ferromagnetic $90^{\circ}$ magnetic domain boundary.

Here we focus on the reorientation of $10 \mathrm{M}$ martensite single crystal in the simplest case of the field-induced

*corresponding author; e-mail: heczko@fzu.cz switching from the single variant to other single variant. This allows probing the magnetization process connected with the nucleation and twin movement in greater details and we can relate the magnetization process to the magnetic domains pattern.

On the other hand, the existence of MIR or the fieldinduced change of the crystal structure orientation offers a unique chance to study different magnetic configurations in single sample, i.e. in the exactly same material. We investigate magnetic domains patterns for all three possible orientations of easy magnetization axis. We demonstrate that the domains size scales with the sample dimension along easy magnetization axis and the domains penetrate through the whole sample.

\section{Experimental methods}

The cuboid single crystal of $\mathrm{Ni}_{50} \mathrm{Mn}_{28.5} \mathrm{Ga}_{21.5}$ (atomic\%) was cut approximately along the $\{100\}$ planes of the parent austenite phase. The size was $5.14 \mathrm{~mm} \times$ $2.53 \mathrm{~mm} \times 0.93 \mathrm{~mm}$ in a single variant state with $c$ axis along the long sample axis. The transformation temperature from cubic austenite to five-layered modulated martensite (10M previously called also $5 \mathrm{M})$ was about $320 \mathrm{~K}$ and the Curie point $T_{\mathrm{C}}=373 \mathrm{~K}$. The structure of martensite is approximated by slightly monoclinic lattice keeping the orientation of cubic austenite. The short $c$-axis of monoclinic lattice is an easy magnetization axis. Calculated demagnetization factors for cuboids sample [6] were $0.12,0.25$, and 0.63 . Magnetization curves were measured by magnetic sample magnetometer PAR up to $1.8 \mathrm{~T}$. All experiments were done at room temperature.

Magnetic domain pattern was investigated using polarized light and magneto-optical indicator film (MOIF) in optical microscope Zeiss. If MOIF is freely put on the ferromagnetic sample and it visualizes the stray field occurring from underlining magnetic domain structure $[7,8]$.

\section{Results and discussion}

Figure 1 shows the magnetization curve of $\mathrm{Ni}-\mathrm{Mn}-\mathrm{Ga}$ single crystal in a single macroscopic variant state. Inset 
shows the initial configuration and sample orientation. The crystal $c$-axis of the variant which is also easy axis of magnetization was initially perpendicular to the magnetic field. In increasing magnetic field the magnetization rotation initially takes place. At about $0.5 \mathrm{~T}$ field the nucleation and then motion of twin boundary occurs and the variant crystal orientation is switched to $c$-axis along the field resulting in rapid magnetization increase. The reorientation is sudden in this case indicating that the nucleation requires larger field than following twin boundary movement $[1,5]$. Additionally, the growth of the variant with $c$-axis along the field decreases the demagnetization of this variant and consequently the field needed for reorientation decreases [9]. In larger field the reorientation is completed and the sample contains again a single variant now with $c$-axis along the field. The process gives rise to the typical magnetization curve of a sample with MIR effect. It is superficially similar to metamagnetic behaviour, however, in this case not only magnetic axis but also structural one is switched. Moreover, the field is much smaller in comparison with usual metamagnetic materials.

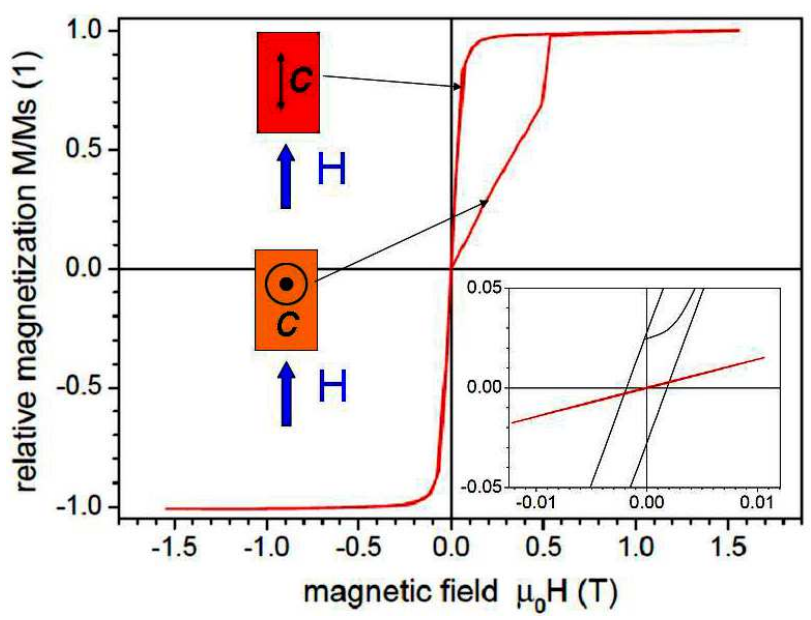

Fig. 1. Magnetization curve of sample exhibiting MIR. Left inset shows the sample orientation prior and after variant switching. The corresponding part of the magnetization curve is pointed. Right inset shows detail of the minor loops (field range about $0.01 \mathrm{~T}$ ) for both orientations of $c$-axis indicated in the left inset (red perpendicular orientation, black - along the field).

The sample exhibits full MIR above $0.5 \mathrm{~T}$ field. This large field is necessary for the nucleation of macroscopic twin variant with $c$-axis along the field and the formation of the mobile $a-c$ twin boundaries. If mobile $a-c$ twin boundaries are present prior magnetizing process the magnetic field needed for reorientation can be lower down to $0.01 \mathrm{~T}[10,11]$. The completeness of the reorientation can be estimated from the squareness of the return branch of magnetization curve. The deviation from the approach of saturation indicates the presence of other variants. The estimated amount of other variants is less than $0.3 \%$.
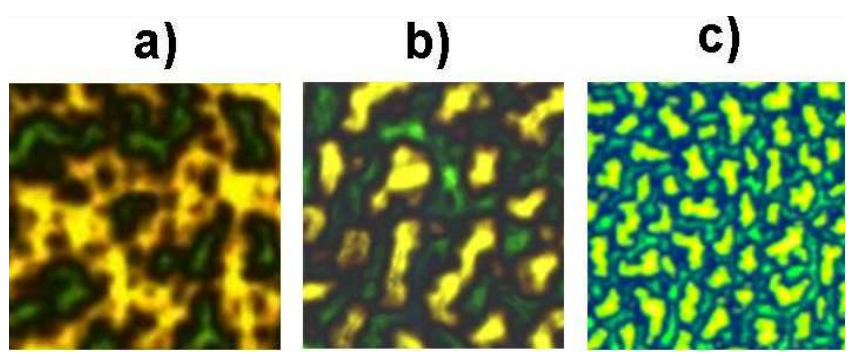

Fig. 2. Optical micrographs of magnetic domain patterns visualized by MOIF for different orientation of $c$ axis, i.e. magnetization easy axis along (a) long, (b) middle and (c) short sample dimension. The size of all
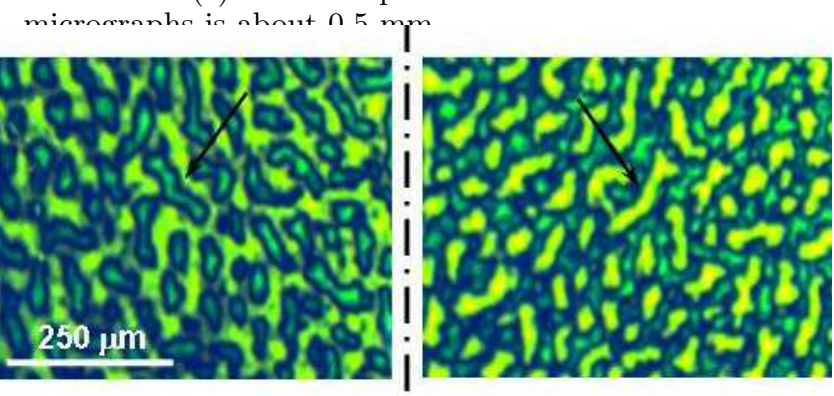

Fig. 3. Magnetic domain patterns on both sides of the sample (top and bottom cut in the same location) with $c$-axis perpendicular to the surface. The symmetry axis marks the folding and pattern mirroring. The arrows are guides for eye pointing selected feature occurring on both sides. The opposite shades of the patterns indicate the different orientations of magnetization (a sink and a well).

The minor loops (inset of Fig. 1) measured prior and after reorientation confirm the described mechanism. Prior switching the loop has no resolvable hysteresis (in vibrating sample magnetometer resolution) which indicates the magnetization rotation process. There is no magnetic domain wall movement. The corresponding domain structure is similar as shown in Figs. 2-4. The minor loop measured after reorientation, i.e. with easy axis along the field, exhibits the hysteresis and the coercive force about $0.002 \mathrm{~T}$. The major loop exhibits only slightly higher value. It is apparent that in this case the magnetization process proceeds by nucleation and the movement of magnetic domain walls in contrast to statement in $[5,12]$. The small value of the hysteresis in comparison with the large magnetic anisotropy (which can be estimated from initial curve in Fig. 1) is surprising. It shows that the nucleation of the new magnetic domains is quite easy process, which can be ascribed to the sharp edges of the sample and underlining twinned microstructure. Even in the single macroscopic variant there are other types of twinning as monoclinic twinning and $a-b$ laminate twinning due to monoclinic lattice [13]. These twins are not affected by magnetic field as all these mesoscopic or microscopic twin domains have the same energy in the magnetic field. However, the intersections of these twins and surface relief due to twinning may serve as 


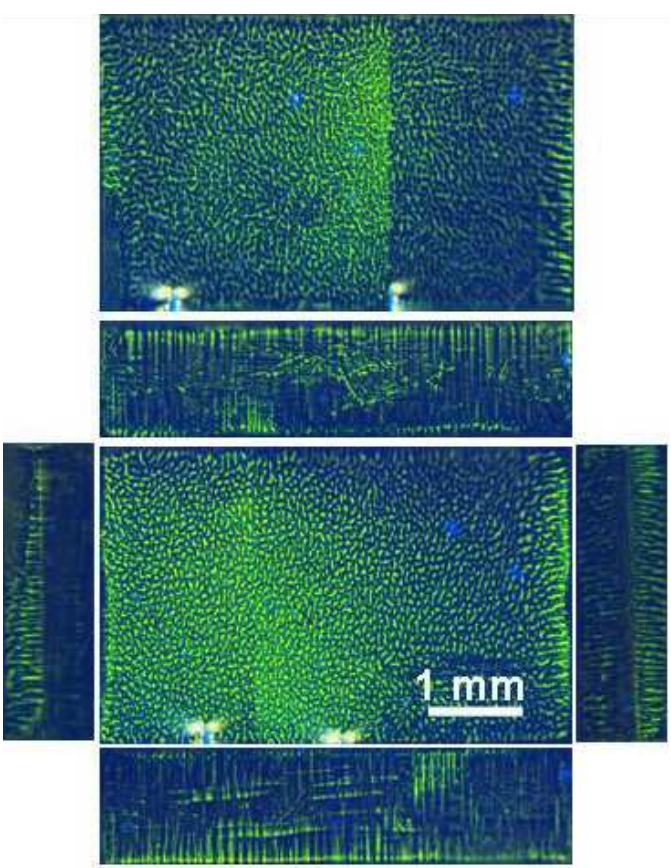

Fig. 4. Distributed 3D picture of domains patterns on all sides of the cuboid sample visualized by MOIF (can be folded). The short edges are not visible sufficiently well due to difficulty to electropolish all sides simultaneously. On the thin longer sides the regular band domain pattern (vertical lines) is disturbed by the presence of the scratches. The picture of the large faces is composed from the several frames which caused variable shading.

nucleation centres. On the other hand, there is no significant pinning of the domain walls in comparison with operational pinning of twin boundary [14].

Figures 2 and 3 show the magnetic domain structure of single variant with $c$-axis and thus also magnetization vector perpendicular to the surface. The magnetic domain structure is relatively simple showing random labyrinth pattern and provides the strongest contrast as the stray field is large for this high anisotropy material. The observed domains are bulk domains, due to MOIF sensitivity on stray field. As shown by magnetic force microscopy (MFM) the magnetic domains on the surface are much smaller of the order of $1 \mu \mathrm{m}$ due to branching [13].

Thanks to MIR we can obtain all three orientations of the $c$-axis along sides of the cuboid sample. Figure 2 demonstrates how the size of the domains scales with the dimension $t$ of the sample along $c$-axis. The size of the domains approximately scales with $\sqrt{t}$ in agreement with the Landau-Kittel model. These bulk domains penetrate whole thickness of the sample as demonstrated in Fig. 3 showing both faces of the sample. The top and bottom faces exhibit mirrored patterns with dark and light contours exchanged indicating opposite orientation of magnetization. This is similar as observed mirroring across $a-c$ twin boundary [15].

In order to obtain the full picture we investigated the magnetic domains pattern on all six faces of the sample in single variant state. Figure 4 shows the result. To obtain the $3 \mathrm{D}$ visualization of the domains, the micrographs can be folded. The figures show that the magnetic domains pattern is relatively regular and well established despite the various scratches on the surface. This insensitivity demonstrates that the visualized domain pattern originated from the volume. The band domains on the perpendicular faces run across whole area in agreement with observation shown in Fig. 3. On the edges there is apparent slight magnetization misorientation due to magnetostatic field.

\section{Conclusions}

Magnetization process in single variant is correlated with magnetic domain structure. The MOIF is good method to observe bulk domains, which are decisive in magnetization process but its resolution is limited. Thanks to MIR all three orientations of magnetizations in single crystal can be probed. The $3 \mathrm{D}$ visualization of magnetic domains pattern for one orientation is provided.

\section{Acknowledgments}

This work was supported by the project No. 15-00262S of Czech Science Foundation.

\section{References}

[1] O. Heczko, N. Scheerbaum, O. Gutfleisch, in: Nanoscale Magnetic Materials and Applications, Eds. J.P. Liu, E. Fullerton, O. Gutfleisch, D.J. Sellmyer, Springer-Verlag, New York 2009, p. 399.

[2] O. Heczko, A. Sozinov, K. Ullakko, IEEE Trans. Magn. 36, 3266 (2000).

[3] A. Sozinov, N. Lanska, A. Soroka, W. Zou, Appl. Phys. Lett. 102, 021902 (2013).

[4] L. Straka, H. Hanninen, O. Heczko, Appl. Phys. Lett. 98, 141902 (2011).

[5] O. Heczko, IEEE Trans. Magn. 50, 2505807 (2014).

[6] A. Aharoni, J. Appl. Phys. 83, 3432 (1998).

[7] A. Hubert, R. Schäfer, Magnetic Domains: The Analysis of Magnetic Microstructures, SpringerVerlag, Berlin 1998.

[8] A. Neudert, Y.W. Lai, R. Schäfer, M. Kustov, L. Schultz, J. McCord, Adv. Eng. Mater. 14, 601 (2012).

[9] O. Heczko, D. Vokoun, V. Kopecky, M. Beleggia, IEEE Magn. Lett. 6, 1000204 (2015).

[10] O. Heczko, Mater. Sci. Technol. 30, 1559 (2014).

[11] V. Kopecký, O. Perevertov, L. Straka, M. Sevcik, O. Heczko, Acta Phys. Pol. A 128, 754 (2015).

[12] Y.W. Lai, N. Scheerbaum, D. Hinz, O. Gutfleisch, R. Schäfer, L. Schultz, J. McCord, less, Appl. Phys. Lett. 90, 192504 (2007).

[13] O. Heczko, V. Kopecký, L. Fekete, K. Jurek, J. Kopecek, L. Straka, H. Seiner, IEEE Trans. Magn. 51, 2505304 (2015).

[14] O. Heczko, A. Soroka, S.-P. Hannula, Appl. Phys. Lett. 93, 022503 (2008).

[15] Y.-W. Lai, R. Schäfer, L. Schultz, J. McCord, Appl. Phys. Lett. 96, 022507 (2010). 